\title{
Whole-exome sequencing enables correct diagnosis and surgical management of rare inherited childhood anemia
}

\begin{abstract}
Monica Khurana, ${ }^{1,2}$ Donna Edwards, ${ }^{1,2,3}$ Frederic Rescorla, ${ }^{4}$ Caroline Miller, ${ }^{5}$ Ying $\mathrm{He}^{3}$ Elizabeth Sierra Potchanant, ${ }^{3}$ and Grzegorz Nalepa ${ }^{1,2,3,6,7}$

${ }^{1}$ Riley Hospital for Children at IU Health, Indianapolis, Indiana 46202, USA; ${ }^{2}$ Division of Pediatric HematologyOncology, Department of Pediatrics, IU School of Medicine, Indianapolis, Indiana 46202, USA; ${ }^{3}$ Wells Center for Pediatric Research, IU School of Medicine, Indianapolis, Indiana 46202, USA; ${ }^{4}$ Department of Surgery, IU School of Medicine, Indianapolis, Indiana 46202, USA; ${ }^{5}$ Electron Microscopy Core, IU School of Medicine, Indianapolis, Indiana 46202, USA; ${ }^{6}$ Department of Biochemistry, IU School of Medicine, Indianapolis, Indiana 46202, USA; ${ }^{7}$ Department of Medical and Molecular Genetics, IU School of Medicine, Indianapolis, Indiana 46202, USA
\end{abstract}

\begin{abstract}
Correct diagnosis of inherited bone marrow failure syndromes is a challenge because of the significant overlap in clinical presentation of these disorders. Establishing right genetic diagnosis is crucial for patients' optimal clinical management and family counseling. A nondysmorphic infant reported here developed severe transfusion-dependent anemia and met clinical criteria for diagnosis of Diamond-Blackfan anemia (DBA). However, whole-exome sequencing demonstrated that the child was a compound heterozygote for a paternally inherited pathogenic truncating variant (SPTA $1^{\mathrm{c} .4975 \mathrm{C}>\mathrm{T})}$ and a novel maternally inherited missense variant of uncertain significance (SPTA $1^{c .5029 \mathrm{G}>A}$ ) within the spectrin gene, consistent with hereditary hemolytic anemia due to disruption of red blood cell (RBC) cytoskeleton. Ektacytometry demonstrated abnormal membrane flexibility of the child's RBCs. Scanning electron microscopy revealed morphological aberrations of the patient's RBCs. Both parents were found to have mild hereditary elliptocytosis. Importantly, patients with severe RBC membrane defects may be successfully managed with splenectomy to minimize peripheral destruction of misshapen RBCs, whereas patients with DBA require lifelong transfusions, steroid therapy, or hematopoietic stem cell transplantation. As suggested by the WES findings, splenectomy rendered our patient transfusion-independent, improving the family's quality of life and preventing transfusion-related iron overload. This case illustrates the utility of whole-exome sequencing in clinical care of children with genetic disorders of unclear presentation.
\end{abstract}

\section{Ontology terms: anemic pallor; congenital hemolytic anemia; microspherocytosis; \\ reticulocytopenia; unconjugated hyperbilirubinemia}

Published by Cold Spring Harbor Laboratory Press

doi: $10.1101 / \mathrm{mcs} . \mathrm{a} 003152$

\section{INTRODUCTION}

Diagnosis of inherited bone marrow failure syndromes is remarkably difficult because of the striking clinical heterogeneity of these rare diseases. Many patients lack a clear physical or laboratory profile to guide expert clinicians toward a specific single-gene test (Nalepa and Clapp 2018). Therapies required in the setting of acute illness may cloud the ability to pursue diagnostic tests at a later time-for example, blood transfusions may suppress abnormal hematopoiesis and alleviate hemolysis in congenital hemolytic anemia. Nevertheless, it is 
ultimately essential to establish a diagnosis to recommend management and evidencebased counseling for families affected with these uncommon genetic disorders. Therefore, whole-exome sequencing (WES) is increasingly utilized for unbiased clinical diagnostics in individuals whose symptoms are suggestive of a hereditary disease but not sufficiently specific for targeted gene sequencing (Biesecker and Green 2014).

When WES reveals variants of unknown significance (VUS), functional evaluation of the resulting protein changes corroborates the diagnostic findings and may offer clinically useful insights into disease pathogenesis. Ultimately, this approach may unravel unexpected therapeutic options for the affected family. Here, we discuss a child affected with a misdiagnosed genetic disorder, in which WES supported by experimental validation of novel genomic variants established the correct diagnosis and inspired major changes to the proband's and family's clinical management, eliminating the need for chronic red blood cell (RBC) transfusions.

\section{RESULTS}

A nondysmorphic Caucasian infant female was referred to us for management of suspected DBA based on transfusion-dependent anemia since the age of 2 mo when she presented with a severe anemia (hemoglobin $3.2 \mathrm{~g} / \mathrm{dl}$ ) and absolute reticulocyte count of $98 \mathrm{~K} / \mathrm{mm}^{3}$, which was inappropriately low for the degree of anemia (Buttarello 2016). DBA is a clinically heterogeneous disorder, and consensus diagnostic criteria for "classical" DBA include age of $<1 \mathrm{yr}$ at the time of anemia onset, low reticulocyte count, lack of other significant cytopenias, and decreased erythrocytic precursors without other major abnormalities in the bone marrow. A positive family history and the presence of mutations within known DBA genes are considered major supporting diagnostic criteria in this complex syndrome; congenital anomalies, elevated adenosine deaminase (ADA), and fetal hemoglobin (Hgb F) represent further minor supporting criteria for DBA (Vlachos et al. 2008). The patient's family history was reported as normal. Her ADA, pyruvate kinase (PK) activity, and fetal hemoglobin $(\mathrm{HgbF})$ were not tested before the first transfusion. Her bone marrow evaluation was nondiagnostic as specimen dilution prevented quantification of erythroid precursors. There was no jaundice concerning for hemolytic anemia. Given the unclear clinical picture, we considered atypical DBA or another inherited disorder of hematopoiesis. As steroids improve erythropoiesis in DBA and render most patients transfusion-independent (Ball 2011), we pursued a trial of prednisone (2 mg/kg PO daily) and observed an initial hemoglobin increase to $9.4 \mathrm{~g} / \mathrm{dl}$ followed by a drop to $5.7 \mathrm{~g} / \mathrm{dl}$ within a month; therefore, we classified the patient as steroid nonresponsive and discontinued her prednisone. Her DBA-focused sequencing panel and copy-number variation microarray were normal.

The patient required monthly RBC transfusions and developed one hemolytic episode associated with viral illness, resulting in pallor, mild hyperbilirubinemia, and, unexpectedly for presumptive diagnosis of DBA, mild reticulocytosis. We decided to pursue whole-exome sequencing (WES) of the child and her parents to determine the potential underlying inherited cause of her clinical course and allow genetic counseling.

WES of the family revealed that the patient is a compound heterozygote for two erythrocytic $\alpha$-spectrin (SPTA1) variants, consistent with hereditary pyropoikilocytosis (Table 1). The first truncating SPTA1 variant inherited from the father (c.4975C>T; p.R1659X) had been reported in hereditary pyropoikilocytosis (Tolpinrud et al. 2008). The second maternally inherited SPTA1 variant resulted in a novel missense substitution (c.5029G >A; p.G1677R). This variant affects a conserved spectrin domain outside of the spectrin self-association site and is predicted to be deleterious by PROVEAN and PolyPhen-2 algorithms. Neither SPTA1 genetic variant was observed in approximately 6000 individuals of European and African 
Table 1. Genomic findings

\begin{tabular}{lcccccc}
\hline Gene & Genomic location & HGVS cDNA & HGVS protein & Zygosity & Parent of origin & Variant interpretation \\
\hline SPTA1 & Chromosome 1, NC_000001.11 & c.4975 C>T & p.R1659X & Heterozygous & Father & Pathogenic \\
SPTA1 & Chromosome 1, NC_000001.11 & c.5029G >A & p.G1677R & Heterozygous & Mother & Variant of unknown significance \\
\hline
\end{tabular}

American ancestry in the NHLBI Exome Sequencing Project, indicating these are not common benign variants in these populations. In retrospect, both parents had a history of mild unexplained anemia with occasional peripheral elliptocytes and the father had chronic hyperbilirubinemia suggestive of hemolysis, consistent with a diagnosis of mild hereditary elliptocytosis (Gallagher 2005).

Review of the patient's pretransfusion smear (Fig. 1A) combined with scanning electron microscopy (Fig. 1B) revealed microspherocytes, anisopoikilocytosis, and budding RBCs, consistent with congenital hemolytic anemia due to $\alpha$-spectrin deficiency resulting in dysfunctional RBC membrane cytoskeleton. The patient's RBC membrane protein electrophoresis showed the presence of $\beta$-spectrin, whereas $\alpha$-spectrin was nearly undetectable (Fig. 1C). Ektacytometry, performed 2 mo after transfusion, detected abnormal RBC flexibility (Fig. 1D). Elliptocytes and occasional spherocytes were found on both parents' peripheral blood smears, consistent with the diagnosis of hereditary elliptocytosis. Given these genetic findings and ongoing transfusion needs, the child underwent partial laparoscopic splenectomy removing the distal $85 \%$ of the spleen, which resulted in transfusion independence for $12 \mathrm{mo}$ (her transfusion threshold was $7 \mathrm{~g} / \mathrm{dl}$ ). However, the patient's transfusion dependence recurred with hemoglobin nadir of $5.9 \mathrm{~g} / \mathrm{dl}$, correlating with regrowth of her spleen tissue reported on abdominal ultrasound. The patient next underwent full laparoscopic splenectomy. She remains transfusion-independent with normal hemoglobin more than 6 mo postsplenectomy.

\section{DISCUSSION}

Our patient initially received the clinical diagnosis of suspected DBA based on the severe transfusion-dependent anemia with reticulocytopenia starting in infancy. DBA represents a group of genetically distinct ribosomopathies that manifest hematologically as severely impaired erythropoiesis, although it is not clear how genetic impairment of the ribosome selectively arrests RBC production and produces a wide variety of systemic manifestations that vary between patients (Ruggero and Shimamura 2014; Vlachos et al. 2014). Lack of dysmorphic features did not exclude this possibility as roughly half of DBA patients are free of gross malformations (Da Costa et al. 2017). Our patient's ADA activity and hemoglobin $F$ (HgbF) were not tested before her first transfusion and referral to our center. Moreover, we initially did not consider an underlying hemolytic anemia as the cause of her transfusion dependency as she was not jaundiced and did not have gross anipoikilocytosis on her posttransfusion peripheral blood smears, likely because her own erythropoiesis was significantly suppressed by monthly transfusions. However, our retrospective review of her previously inaccessible pretransfusion blood smear revealed anisopoikilocytosis that should have raised the concern of an underlying RBC membrane disorder. These diagnostic challenges highlight the importance of early consultation and provision of original blood and marrow specimens to pediatric hematologists with expertise in inherited bone marrow failure syndromes.

Consistent with a structurally and functionally abnormal RBC membrane cytoskeleton, we observed microspherocytes and bizarrely shaped RBCs on patient's posttransfusion 


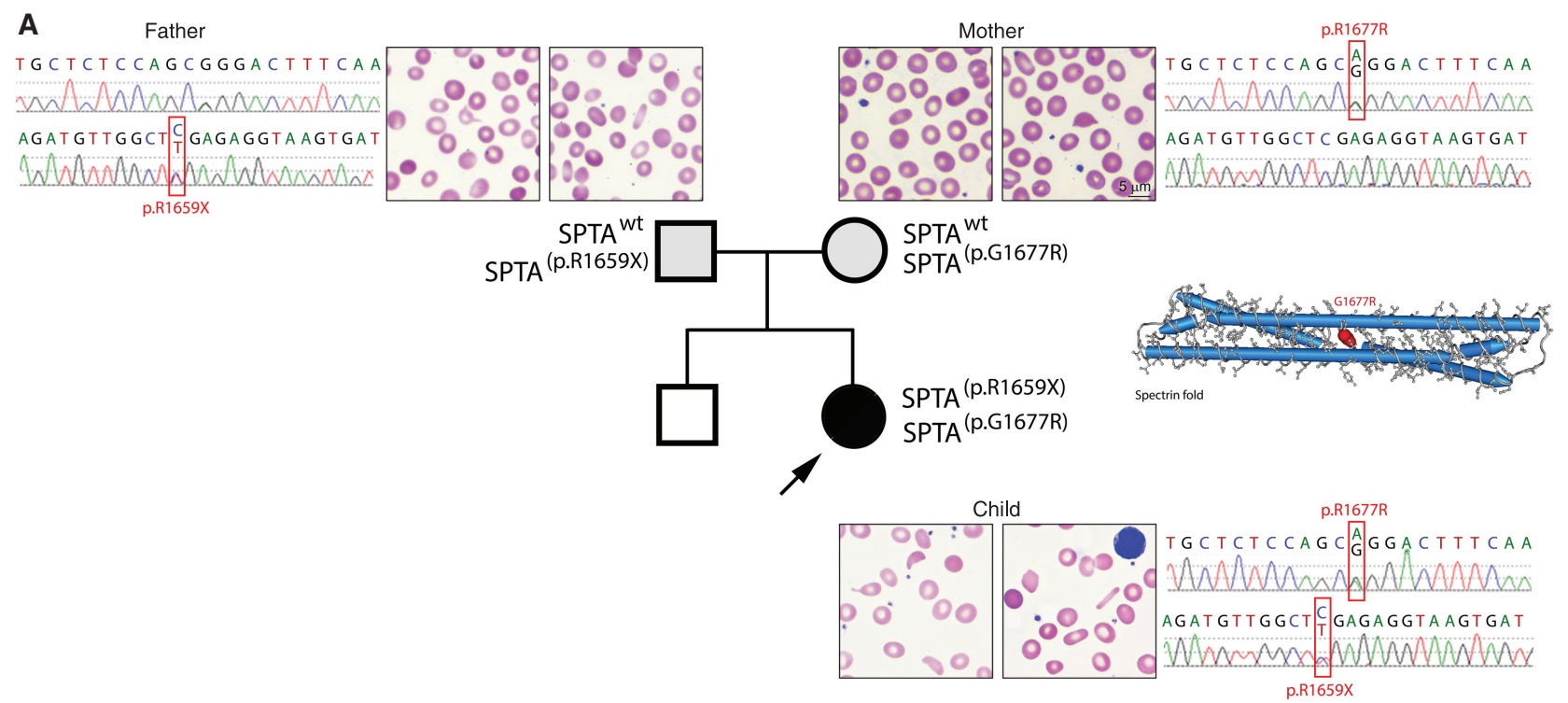

B

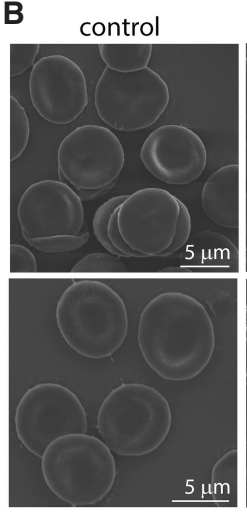

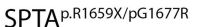

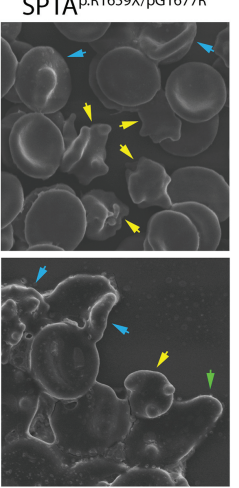

C

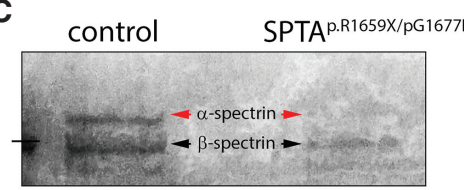

D

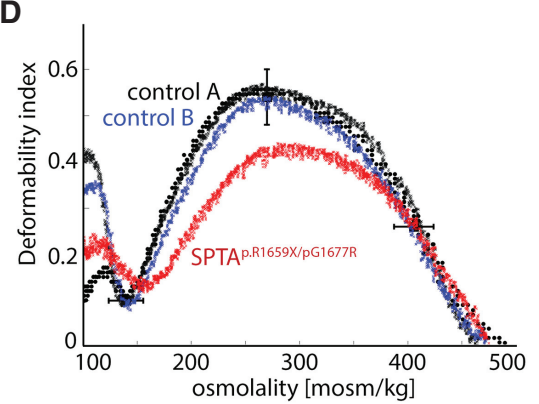

Figure 1. Red blood cell (RBC) membrane protein deficiency identified via whole-exome sequencing. (A) Compound heterozygous SPTA1 mutations identified via whole-exome sequencing in a child with transfusion-dependent anemia. Note anisopokilocytosis, microcytes, and elliptocytes on the peripheral blood smear. Elliptocytes in parents' peripheral smears are consistent with diagnosis of hereditary elliptocytosis. (Insert) Location of the new SPTA1 variant within the spectrin fold. Arrow indicates the affected proband. Healthy brother (white square) was not genetically tested. Mother and father are represented by gray circle and square, respectively. Severity of phenotype in the family members is reflected by grayscale gradient. (B) Microspherocytes (yellow arrows), elliptocyte (green arrow), and RBC with membrane extrusions (blue arrows) visualized via scanning electron microscopy in peripheral blood of the patient compared to healthy control. (C) Decreased $\alpha$-spectrin protein in the index patient's RBCs. (D) Decreased RBC deformability in the index patient.

RBC scanning electron microscopy but not in the blood of healthy controls processed and imaged in the same manner (Fig. 1B). Notably, the simplified approach to RBC scanning electron microscopy we have developed for this work produced high-quality RBC images, suggesting the potential utility of this technically easy strategy to examine RBC structure in more detail compared to conventional microscopy. Ektacytometry confirmed abnormal deformability of the patient's RBCs resulting from the biochemically validated deficiency of RBC membrane-associated $\alpha$-spectrin in this child (Fig. 1C,D); in fact, it likely underestimated the severity of RBC membrane defect because these experiments were done 2 mo post-RBC transfusion and therefore evaluated a mixture of normal and 
COLD SPRING HARBOR Molecular Case Studies
From WES to surgery to diagnose and treat anemia

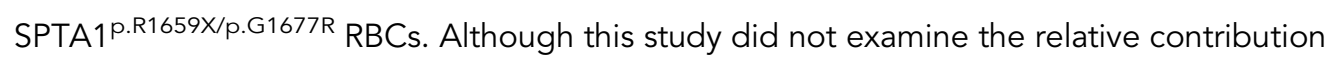
of the paternal (p.R1659X) and maternal (p.G1677R) mutation, we hypothesize the paternal mutation is more severe as it truncates the protein and the father had a known mild borderline hemolysis.

Different pathophysiology of DBA and RBC membrane disorders provides distinct therapeutic opportunities. In DBA, anemia is caused by impaired RBC production. Steroids rescue erythropoiesis in $>50 \%$ of DBA patients; others require RBC transfusions for their lifetimes or hematopoietic stem cell transplantation (Vlachos and Muir 2010). Long-term care of these complex patients requires aggressive management of toxic iron overload resulting from chronic transfusions, side effects of chronic steroid use, as well as cancer surveillance. Contrastingly, in hereditary RBC membranopathies, anemia is caused by elimination of misshapen RBCs by spleen macrophages beyond the bone marrow's ability to compensate for hemolysis. Therefore, splenectomy provides a viable surgical option to manage severe RBC membrane disorders. However, the potential benefits of decreased transfusion dependency must be balanced with the long-term risk of overwhelming postsplenectomy infections (OPSIs) (Jugenburg et al. 1999; Edgren et al. 2014) requiring immunizations against encapsulated bacteria as well as prophylactic penicillin (Davies et al. 2011). Subtotal splenectomy may alleviate transfusion dependency in severe hereditary spherocytosis (Bader-Meunier et al. 2001; Buesing et al. 2011) and neonatal pyropoikilocytosis (Medejel et al. 2008) without increasing the risk of OPSIs and thrombosis. Some (Medejel et al. 2008) but not all (Rice et al. 2003; Buesing et al. 2011) children with hemolytic anemia post-partial splenectomy require full splenectomy at a later time. Therefore, the risks and benefits of splenectomy in children with RBC membrane disorders should be carefully examined on a personalized basis.

In our patient, we decided to initially proceed with partial splenectomy because of the patient's young age. Although this approach provided a temporary decrease in transfusion needs, our patient ultimately required a complete splenectomy $>12$ mo later as her transfusion-dependent anemia reoccurred, presumably because of increased RBC destruction in the expanding remainder splenic tissue. Total splenectomy eliminated our patient's transfusion dependence, halting her transfusion-related iron overload and exposure to blood products, minimizing her need for clinic visits. The diagnosis not only allowed genetic counseling for the family but also provided information relevant to the parents' health maintenance, including their risk of chronic hemolysis and cholelithiasis due to chronic subclinical hemolysis. Interestingly, the child's father had been undergoing evaluation for suspected Gilbert's syndrome at another facility because of mild asymptomatic hyperbilirubinemia, which we now believe is due to his chronic hemolysis secondary to his SPTA $1^{\text {R1659X }}$ truncation.

WES is not free of limitations. First, this diagnostic technique will not identify pathogenic mutations located outside of the exome and may not capture large DNA deletions or duplications (Nalepa and Clapp 2018). Second, identification of VUS may cause unique challenges as detailed functional analysis of VUSs, as reported in this study, is not always possible. However, this case illustrates the clinical utility of WES in children with rare congenital disorders that have unclear clinical presentation and/or laboratory profile (Sharma and Nalepa 2016).

\section{METHODS}

\section{Whole-Exome Sequencing}

Patients' and parents' diagnostic whole-exome sequencing of nuclear and mitochondrial DNA was done at GeneDx. Using genomic DNA from the submitted specimen, the Agilent 
Table 2. Whole-exome sequencing metrics

\begin{tabular}{ll}
\hline Mean depth of coverage & $111 \times$ \\
Percentage of exome with at least 10x coverage & $96.1 \%$ \\
\hline
\end{tabular}

Clinical Research Exome kit was used to target the exonic regions and flanking splice junctions of the genome. These targeted regions were sequenced simultaneously by massively parallel (NextGen) sequencing on an Illumina HiSeq 2000 sequencing system with 100-bp paired-end reads. Bidirectional sequence was assembled, aligned to reference gene sequences based on human genome build GRCh37/UCSC hg19, and analyzed for sequence variants using a custom-developed analysis tool (Xome Analyzer). Capillary sequencing or another appropriate method was used to confirm all potentially pathogenic variants identified in this individual and relative samples. Sequence alterations were reported according to the Human Genome Variation Society (HGVS) nomenclature guidelines. Mean depth of coverage (mean number of sequence reads obtained across the whole exome; specifically, the coding exons and splice junctions of protein-coding RefSeq genes that are captured by the Agilent Clinical Research Exome kit) was 111x. Additionally, the entire mitochondrial genome from each sample was amplified and sequenced using a solid-state sequencing by synthesis process. DNA sequence was assembled and analyzed in comparison with the revised Cambridge Reference Sequence ( $\mathrm{rCRS}$ ) and the reported variants and polymorphisms listed in the MITOMAP database (http://www.mitomap.org). The initial DBA next-gen panel (RPS19, RPL5, RPS10, RPL11, RPL35A, RPS26, RPS24, RPS17, RPS7, RPL19, and RPL26) was done at Ambry Genetics. See Table 2 for the WES metrics.

\section{RBC Evaluation}

For scanning electron microscopy, blood samples in fixative (2\% paraformaldehyde/2\% glutaraldehyde in phosphate buffer) were air-dried on silicon wafers overnight. Then gold-palladium sputter coating specimens were viewed and imaged with a JEOL, LV 6390 (Peabody) Scanning Electron Microscope. Peripheral blood smear images were acquired on a Zeiss Axio Lab.A1 microscope with an Axiocam 105 color camera and processed with ZEN (Carl-Zeiss) and Imaris (Bitplane) software. Osmotic gradient ektacytometry was performed and interpreted at the RBC Laboratory, Children's Oakland Research Institute, UCSF Benioff's Children's Hospital, Oakland, CA. Specimen was shipped overnight in room temperature and accompanied by healthy travel control to ensure results are not affected by shipping.

\section{ADDITIONAL INFORMATION}

\section{Data Deposition and Access}

The variants were submitted to ClinVar (http://www.ncbi.nlm.nih.gov/clinvar/) and can be found under accession numbers SCV000804855.1 and SCV000804576. Patient consent was not obtained for deposition of raw sequencing data.

\section{Ethics Statement}

Informed consent was obtained from the proband's parents. Clinical specimen biobanking was approved by the IU School of Medicine Institutional Review Board under the Indiana Pediatric Biobank protocol (IRB \#1501467439). 
Competing Interest Statement

The authors have declared no competing interest.

Received May 7, 2018; accepted in revised form August 7, 2018.

\section{Acknowledgments}

We thank the family for agreeing to participate in this study. We appreciate discussions with Dr. Jane Juusola and Meredith Strait (both GeneDx). The DBA next-gen panel was done at Ambry Genetics. RBC ektacytometry was done and analyzed at Children's Hospital Oakland Research Institute (Oakland, CA) using two healthy controls.

\section{Author Contributions}

M.K. and G.N. designed the study, performed experiments, analyzed the data, and provided clinical care to the family. D.E., Y.H., and E.S.P. performed the experiments and analyzed the data. C.M. performed the scanning electron microscopy imaging. G.N. wrote and all authors edited and approved the manuscript.

\section{Funding}

Research in the Nalepa laboratory is supported by the National Institutes of Health (NIH) R01-HL132921-01A1, St. Baldrick's Scholar Award, The Heroes Foundation, Riley Children's Foundation, and The Precision Genomics internal funds.

\section{REFERENCES}

Bader-Meunier B, Gauthier F, Archambaud F, Cynober T, Miélot T, Dommergues JP, Warszawski J, Mohandas N, Tchernia G. 2001. Long-term evaluation of the beneficial effect of subtotal splenectomy for management of hereditary spherocytosis. Blood 97: 399-403.

Ball S. 2011. Diamond Blackfan anemia. Hematology Am Soc Hematol Educ Program 2011: 487-491.

Biesecker LG, Green RC. 2014. Diagnostic clinical genome and exome sequencing. N Engl J Med 370: $2418-2425$.

Buesing KL, Tracy ET, Kiernan C, Pastor AC, Cassidy LD, Scott JP, Ware RE, Davidoff AM, Rescorla FJ, Langer JC, et al. 2011. Partial splenectomy for hereditary spherocytosis: a multi-institutional review. J Pediatr Surg 46: $178-183$.

Buttarello M. 2016. Laboratory diagnosis of anemia: are the old and new red cell parameters useful in classification and treatment, how? Int J Lab Hematol 38: 123-132.

Da Costa L, O'Donohue MF, van Dooijeweert B, Albrecht K, Unal S, Ramenghi U, Leblanc T, Dianzani I, Tamary $H$, Bartels $M$, et al. 2017. Molecular approaches to diagnose Diamond-Blackfan anemia: the EuroDBA experience. Eur J Med Genet doi: 10.1016/j.ejmg.2017.10.017.

Davies JM, Lewis MP, Wimperis J, Rafi I, Ladhani S, Bolton-Maggs PH; British Committee for Standards in Haematology. 2011. Review of guidelines for the prevention and treatment of infection in patients with an absent or dysfunctional spleen: prepared on behalf of the British Committee for Standards in Haematology by a working party of the Haemato-Oncology task force. Br J Haematol 155: 308-317.

Edgren G, Almqvist R, Hartman M, Utter GH. 2014. Splenectomy and the risk of sepsis: a population-based cohort study. Ann Surg 260: 1081-1087.

Gallagher PG. 2005. Red cell membrane disorders. Hematology Am Soc Hematol Educ Program 13-18. doi: 10.1182/asheducation-2005.1.13

Jugenburg M, Haddock G, Freedman MH, Ford-Jones L, Ein SH. 1999. The morbidity and mortality of pediatric splenectomy: does prophylaxis make a difference? J Pediatr Surg 34: 1064-1067.

Medejel N, Garçon L, Guitton C, Cynober T, Bader-Meunier B. 2008. Effect of subtotal splenectomy for management of hereditary pyropoikilocytosis. Br J Haematol 142: 315-317.

Nalepa G, Clapp DW. 2018. Fanconi anaemia and cancer: an intricate relationship. Nat Rev Cancer 18: 168-185.

Rice HE, Oldham KT, Hillery CA, Skinner MA, O'Hara SM, Ware RE. 2003. Clinical and hematologic benefits of partial splenectomy for congenital hemolytic anemias in children. Ann Surg 237: 281-288.

Ruggero D, Shimamura A. 2014. Marrow failure: a window into ribosome biology. Blood 124: 2784-2792.

Sharma R, Nalepa G. 2016. Evaluation and management of chronic pancytopenia. Pediatr Rev 37: 101-111; quiz 112-103. 
Tolpinrud W, Maksimova YD, Forget BG, Gallagher PG. 2008. Nonsense mutations of the $\alpha$-spectrin gene in hereditary pyropoikilocytosis. Haematologica 93: 1752-1754.

Vlachos A, Muir E. 2010. How I treat Diamond-Blackfan anemia. Blood 116: 3715-3723.

Vlachos A, Ball S, Dahl N, Alter BP, Sheth S, Ramenghi U, Meerpohl J, Karlsson S, Liu JM, Leblanc T, et al. 2008.

Diagnosing and treating Diamond Blackfan anaemia: results of an international clinical consensus conference. Br J Haematol 142: 859-876.

Vlachos A, Blanc L, Lipton JM. 2014. Diamond Blackfan anemia: a model for the translational approach to understanding human disease. Expert Rev Hematol 7: 359-372. 


\section{COLD SPRING HARBOR Molecular Case Studies}

\section{Whole-exome sequencing enables correct diagnosis and surgical management of rare inherited childhood anemia}

Monica Khurana, Donna Edwards, Frederic Rescorla, et al.

Cold Spring Harb Mol Case Stud 2018, 4: a003152

Access the most recent version at doi: $10.1101 / \mathrm{mcs} . a 003152$

References

License

Email Alerting Service
This article cites 19 articles, 6 of which can be accessed free at: http://molecularcasestudies.cshlp.org/content/4/5/a003152.full.html\#ref-list-1

This article is distributed under the terms of the Creative Commons Attribution-NonCommercial License, which permits reuse and redistribution, except for commercial purposes, provided that the original author and source are credited. Receive free email alerts when new articles cite this article - sign up in the box at the top right corner of the article or click here. 